\title{
Diffuse Alveolar Hemorrhage after Clopidogrel Use
}

\author{
Tolga Onuk, Göktürk İpek, Mehmet Baran Karataş, Recep Hac1, Neşe Çam
}

Department of Cardiology, Dr. Siyami Ersek Cardiothoracic and Vascular Surgery Center, İstanbul, Turkey

Diffuse alveolar hemorrhage (DAH) is a life-threatening disorder due to impairment of the alveolar-capillary basement membrane resulting from injury or inflammation of the arterioles, venules or alveolar septal capillaries (1). Several autoimmune diseases, infections, drugs and toxins are associated with the development of DAH (2). The diagnosis of DAH is based on clinical suspicion with laboratory, radiologic and pathologic findings. Early diagnosis is crucial for early treatment and survival.

A 57-year old male patient presented to the emergency department with sudden onset of chest pain and shortness of breath. His medical history was remarkable for diabetes mellitus and hypertension. In the emergency department, he had a heart rate of 110 beats/min and a blood pressure of $80 / 50$ $\mathrm{mmHg}$. There was no evidence of heart failure. Acute anterior myocardial infarction was diagnosed based upon the results of 12-lead electrocardiography showing ST-segment elevation in leads V1 through V6. Emergency coronary angiogram was performed after the patient had received $300 \mathrm{mg}$ aspirin (Bayer; Leverkusen, Germany) and $600 \mathrm{mg}$ clopidogrel (Sanofi; Gentilly, France) treatment but glycoprotein $2 \mathrm{~b} / 3 \mathrm{a}$ inhibitors were not used. Total occlusion of proximal left anterior descending artery (LAD) was detected and a $2.75 \times 16 \mathrm{~mm}$ everolimus eluting stent (Xience; Abbott, Illinois, USA) was implanted to the culprit segment and TIMI 3 flow was restored. On the second day after admission the patient developed continuous hemoptysis. Thoracic computed tomographic (CT) scan showed bilateral ground glass opacities in the upper posterior segments and whole lower segments of both lungs (Figure 1). These were attributed to bleeding into the alveolar spaces and the patient was diagnosed as DAH. Patient's symptoms and hemoptysis dramatically improved after discontinuation of clopidogrel. CT revealed regression of DAH on the twelfth day after admission (Figure 2). Written and signed informed approvals were acquired from the patient for the percutaneous procedures and for the documents to be published.

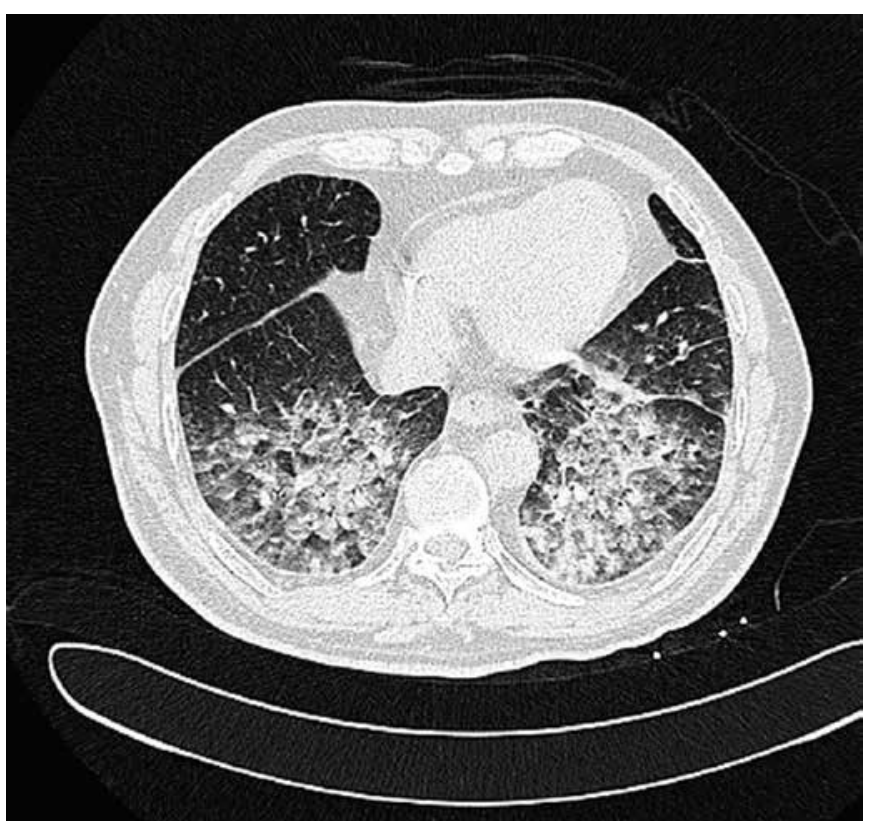

FIG. 1. Chest-computed tomography on the second day after admission shows diffuse alveolar hemorrhage on both lung fields.

Diffuse alveolar hemorrhage is a very important disease with high mortality rates if untreated. Systemic vasculitis such as Wegener granulomatosis, microscopic polyarteritis, Goodpasture's syndrome, connective tissue diseases, infections and drugs should be ruled out if DAH is suspected (3). Early diagnosis and treatment are life-saving in this situation. Cessation of the responsible drug, treatment of infection and reversal of extreme anticoagulation is recommended in treatment. Systemic glucocorticoids and immunosuppressive therapy play an important role in the treatment of inflammatory causes of DAH (4). DAH is a rare complication after antiplatelet treatment in acute coronary syndromes. We have shown DAH due to clopidogrel in this case. This has been shown first by Kilaru et al (5). Clopidogrel is a very potent drug for reducing ischemic events and stent thrombosis after coronary interventions.

Address for Correspondence: Dr. Tolga Onuk, Department of Cardiology, Dr. Siyami Ersek Cardiovascular and Thoracic Surgery Hospital, İstanbul, Turkey 


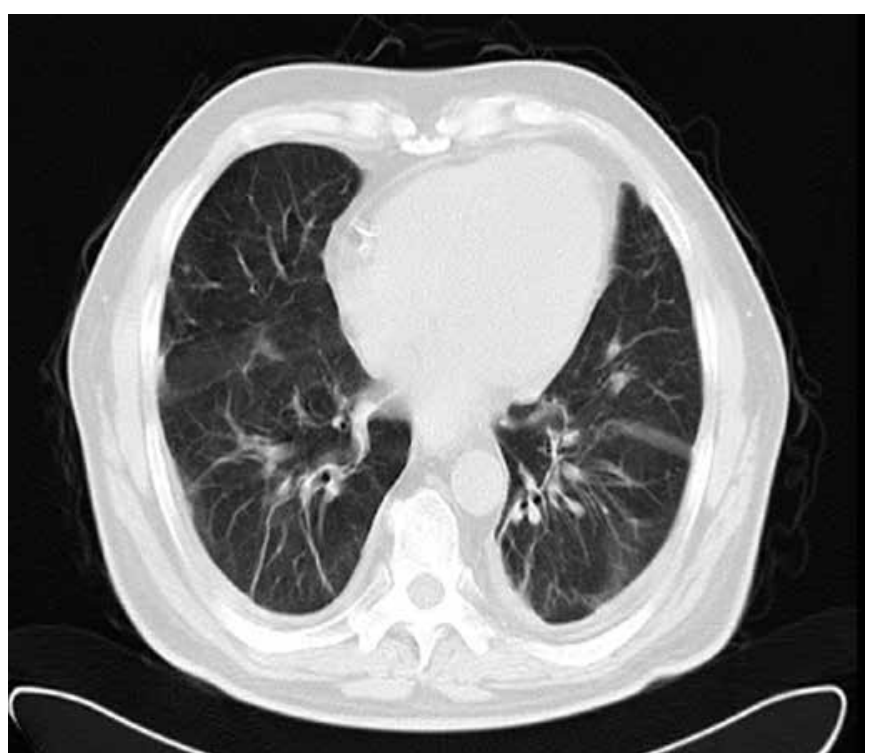

FIG. 2. Chest-computed tomography on the twelfth day after admission shows regression of diffuse alveolar hemorrhage on both lung fields.

Besides that, bleeding complications should be taken into consideration especially in high risk patients.

\section{Ethics Committee Approval: N/A.}

Informed Consent: Informed consent was obtained from the patient who participated in this case.

Peer-review: Externally peer-reviewed.
Author contributions: Concept - T.O.; Design - T.O.; Supervision - T.O.; Resource - G.I.; Materials - B.K.; Data Collection and/ or Processing - R.H.; Analysis and/or Interpretation - T.O.; Literature Search - N.Ç.; Writing - T.O.; Critical Reviews - T.O.

Conflict of Interest: No conflict of interest was declared by the authors.

Financial Disclosure: The authors declared that this study has received no financial support.

\section{REFERENCES}

1. Franks TJ, Koss MN. Pulmonary capillaritis. Curr Opin Pulm Med 2000;6:430-5. [CrossRef]

2. Mark EJ, Ramirez JF. Pulmonary capillaritis and hemorrhage in patients with systemic vasculitis. Arch Pathol Lab Med 1985; 109:413-8.

3. Leatherman JW. The lung in systemic vasculitis. Semin Respir Infect 1988;3:274-88.

4. Raptis A, Mavroudis D, Suffredini A, Molldrem J, Rhee FV, Childs R, et al. High-dose corticosteroid therapy for diffuse alveolar hemorrhage in allogenic bone marrow stem cell transplant recipients. Bone Marrow Transplant 1999;24:879-83. [CrossRef]

5. Kilaru PK, Schweiger MJ, Kozman HA, Weil TR. Diffuse alveolar hemorrhage after clopidogrel use. $J$ Invasive Cardiol 2001;13:535-7. 